\title{
Magnetic coupling of photosphere and corona: MHD simulation for multi-wavelength observations
}

\author{
Jörg Büchner ${ }^{1}$, B. Nikutowski ${ }^{1}$ and A. Otto ${ }^{2}$ \\ ${ }^{1}$ Max-Planck-Institut für Sonnensystemforschung, 37191 Katlenburg-Lindau, Germany \\ email: buechner@linmpi.mpg.de \\ ${ }^{2}$ University of Fairbanks, Alaska, Fairbanks, AK, USA
}

\begin{abstract}
Magnetic coupling relates the energy sources below the photosphere to the structure and dynamics of the chromosphere and corona of the Sun. Multi-wavelength investigations of the solar activity provides the information needed to understand the acting physical mechanisms. Descriptions of magnetic coupling often start with force-free mathematical extrapolations of the photospheric magnetic field. Observations have revealed, however, perpendicular electric current sheets, reconnection and other locally non-force-free structures in chromosphere and corona. Obviously, the solar magnetic coupling includes dynamic forcing due to photospheric plasma motion. We developed a model which though it starts with force-free extrapolated observed photospheric magnetic field additionally takes into account the energy input due to the chromospheric neutral gas and plasma motion. We demonstrate the abilities of our model by applying it to SoHO multi-wavelength observations of an EUV bright point (BP).
\end{abstract}

\section{Introduction}

Magnetic fields do not just passively link the interior and the atmosphere of the Sun. They also determine dynamics and energetics of the corona, heating and particle acceleration which coined the term "magnetic coupling" for the solar atmosphere. Multiwavelength observations are necessary to understand the mechanism of magnetic coupling. Of course, first of all one needs to know the magnetic field. Routinely the magnetic field is observed only at the photospheric level. The forcefree assumption which allows currents only parallel to the magnetic field and no Lorentz forces is usually used to derive coronal fields. Forcefree magnetic fields exclude, however, perpendicular current sheets (Solanki, Lagg, Woch, Krupp \& Collados (2003)), reconnection (Van Driel-Gesztelyi (2003)) and other important magnetic coupling phenomena. Hence plasma models are needed to understand the magnetic coupling between photosphere, chromosphere and corona. There exist static models, semi-empirically fitting temporally and spatially averaged continua and line intensities (Vernazza, Avrett \& Loeser (1981), Fontenla, Avrett, \& Loeser (2002)). Existing dynamic models usually are limited to acoustic effects Carlsson \& Stein (2002). In order to describe the solar atmospheric coupling one has to take into account magnetic fields, and also the photospheric plasma motion which Gudiksen \& Nordlund (2002) described statistically. Different from their approach we have developed a model which directly uses observed photospheric magnetic fields and plasma motion (Büchner, Nikutowski, \& Otto (2004)). In section $\S 2$ we shortly describe our model, in section $\S 3$ we demonstrate its ability applying it to model an EUV-bright point (BP) and in $\S 4$ we discuss the limits of the current model and give an outlook toward its further development adn applicability.

\section{Model}

Our model of the magnetic coupling between photosphere, chromosphere and corona considers the interaction of plasma and neutral gas in the chromosphere, where collisions 
dominate implying ionization and recombination, friction between plasma and neutral gas as well as their thermal contact. We basically solve the coupled MHD-neutral gas equations. The simulation starts with observed longitudinal photospheric magnetic fields which we extrapolate to the corona by solving the forcefree field equation $\nabla \times \mathbf{B}=\alpha \mathbf{B}$. We developed a magnetic field extrapolation based on a particular Fourier expansion which can be used as an initial equilibrium for a MHD simulation. A Fourier expansion requires periodicity in the $x$ and $y$ directions. By choosing a symmetry condition for $B_{z}$ at the $x$ and $y$ boundaries of the system one can construct a periodic domain $-L_{x} \leqslant x \leqslant L_{x}$, $-L_{y} \leqslant y \leqslant L_{y}$ four times as large as the original region $\left(0 \leqslant x \leqslant L_{x}, 0 \leqslant y \leqslant L_{y}\right)$, within which the solution is looked for. The larger region is fully periodic by construction and the total magnetic flux through it is balanced to a high degree. The approach is similar to the one by Seehafer (1978), however, it is constructed to allow well posed, MHD compatible boundary conditions at the boundaries. After determining the initial magnetic configuration based on measured photospheric fields and with the chromospheric and coronal plasma being in a high-plasma- $\beta$ state we than "fill" the initial magnetic field configuration in accordance with the VAL model (Vernazza, Avrett \& Loeser (1981)). In our model we take into account the cross-field plasma motion in the chromosphere as a boundary condition based on observed direction and speed of plasma motion obtained from photospheric magnetograms. Since in the chromosphere neutral gas and plasma are strongly coupled, we impose gas vortices chosen in accordance with the observed photospheric plasma motion as a boundary condition. More details of the simulation odel are given in Büchner et. al, AGU monograpgh on acceleration (in press).

\section{Simulation results}

We applied our simulation model to SoHO EIT and SUMER multi-wavelength observations of an EUV-bright point (BP), by Madjarska, Doyle, Teriaca \& Banerjee (2003), between 15:24 UT and 07:04 UT on October 17/18 1996. During this interval the longitudinal component of the photospheric magnetic field was obtained by SoHO-MDI with a linear resolution of $438 \mathrm{~km}$ (pixel size). The BP was observed above a bipolar magnetic field structure. We show the result of a simulation which started with the magnetic field configuration observed at 01:30 UT on October 18th in an area of $32 \times 32 \mathrm{Mm}^{2}$ including the bipolar magnetic field below the BP. The initial magnetic field configuration was extrapolated using the longitudinal photospheric field component determined by SoHO-MDI. Since no vector magnetic field information was available we assumed $\alpha=0$ at $t=0$, i.e. we started with a potential field configuration. As the boundary condition we derived the cross-field plasma flow velocity from the photospheric displacements of the magnetic flux during the time interval 01:30 UT till 03:00 UT. Two types of motion are characteristic for this time interval: the two opposite-polarity regions approach each other until they fade away (at about 07:00 UT) and one polarity does slightly rotate. The height dependent plasma and neutral gas density is added in accordance with the VAL-model. The initial chromosphere is about 1.5 Mm thick. We choose a non-uniform grid to meet the requirement of having a high plasma - $\beta$ (higher plasma/gas pressure rather than magnetic pressure) at the lower boundary reaching the much lower gaspressurized corona via the relatively thin chromospheric layer, where we enhanced the grid resolution to resolve the chromosphere by at more than 10 grid points. Hence we carried out a simulation on a non-uniform grid with 49 points in the vertical direction $(z)$, and $131 \times 131$ grid points in the horizontal (periodic) $x$ and $y$ directions, including mathematical boundaries. The grid spans a volume of $32 \times 32 \times 15 \mathrm{Mm}^{3}$ excluding the mathematical boundaries, giving a vertical resolution from $150 \mathrm{~km}$ in the chromosphere to $800 \mathrm{~km}$ in the corona and of $500 \mathrm{~km}$ in the horizontal directions. 


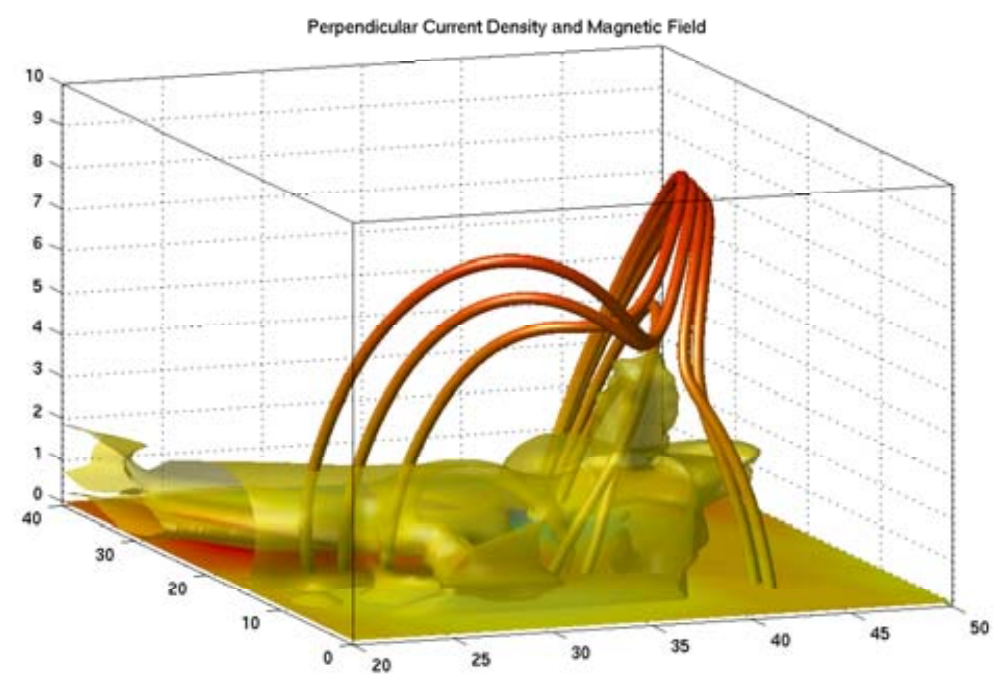

Figure 1. Simulated magnetic field and iso-surface of perpendicular current density above a bipolar magnetic field region which coincides with an observed EUV bright point (BP)

Immediately after the forcing of the plasma by neutral gas motion is started, currents are induced in chromosphere and corona. Already within a few minutes in well localized spots the current density reaches the threshold $\mathbf{j}_{\text {crit }}=0.7$ or $1.4 \mathrm{~mA} \mathrm{~m}^{-2}$, at which the anomalous resistivity switches on. We chose this value by considering an ion-acoustic-type current instability after the current-carrier velocity exceeds the sound speed. Additionally we took into account that simulation threshold must be by about a factor 500 lower than the one calculated from the instability criterion since our spatial (grid) resolution is about 500 times larger than the size of the filamented currents concentrated at kinetic scales typically of the order of $1 \mathrm{~km}$. Figure 1 depicts a iso-surface of a constant current density $\mathbf{j}_{\perp}=0.15$ for the current flow perpendicular to the simulated magnetic field $37 \mathrm{~min}$ after the forcing has started. While most of the simulation domain stays forcefree (no perpendicular currents) also regions of enhanced perpendicular currents appear, mainly in the chromosphere, i.e. below the transition region. The latter is located at about $z=3$ in our normalization. In the chromosphere proper the high collisional resistivity immediately diffuses the currents away. Starting in the upper part of the chromosphere, in the transition region to the corona, however, the built up of the magnetic tension continues until reconnection relaxes the stresses. Although we diagnose the resulting three-dimensional reconnection mainly by its parallel electric fields we also observed the specific topology change, characteristic for this kind of shear flow reconnection. Indeed, in the transition region reconnection begins soon after the photospheric motion has started and continues due to the continued forcing. The corresponding change of the magnetic connection through the portion of the current sheet which reaches the transition region can clearly be seen in Figure 1. The Figure depicts nearby started magnetic field lines bifurcate in the transition region around the current sheet. Since the photospheric motion transports more and more magnetic flux toward the current sheet it permanently supplies energy for the reconnection process (for the animated temporal evolution see http://www.linmpi.mpg.de/english/theorie/tsssp). A comparison with the multi-wavelength observation results of Madjarska, Doyle, Teriaca \& Banerjee (2003) revealed that the simulated current sheet location where magnetic reconnection takes place coincides with the observed bright point position. 


\section{Summary and discussion}

Our simulation model reveals the non-forcefree magnetic coupling between photosphere and corona of the sun for the observed photospheric magnetic fields and plasma motion. Applying it to an EUV-bright point observation of Madjarska, Doyle, Teriaca \& Banerjee (2003) we could show that the plasma flow in the photosphere causes the formation of a localized current sheet in and above the transition region at the position of the EUV-BP. The enhanced current flow makes the current sheet resistive and allows stress relaxation by current dissipation and reconnection which power the BP. Other than the collision of neighboring magnetic loops suggested by Priest, Parnel \& Martin (1994), or with abovelying preexisting fluxes as in Longcope (1998) in our BP-model the currents induced by photospheric motion supply the energy for the energization of the BP.

Although our model reaches beyond static models like Vernazza, Avrett \& Loeser (1981) and Fontenla, Avrett, \& Loeser (2002) and the dynamic, but still acoustic, nonmagnetic, models of Carlsson \& Stein (2002) it is so far limited to the use of a simplified energy equation which does not include radiation transfer, losses and sources. Another limitation comes from the limited spatial resolution of magnetic field observations and simulation. Although the solar corona is not collisionless, the collisional scales such as the inverse magnetic Lundquist number (ratio of magnetic diffusion time over the Alfven time scales) are insufficient to explain the time scale of instabilities for the release of magnetic energy and structure. Since dissipation in space plasma usually occurs on microphysical scales such as Larmor radii or inertial lengths the typical spatial scale resolved in a threedimensional simulation is far too large to form the expected thin filamented boundary layers and current sheets. Nevertheless, MHD simulations provide very reasonable results predicting the evolution of the global system.

Many applications of the proposed modelling method are possible. Based on the photospheric plasma motion inferred from MDI observations the simulation predicts the critical regions of current sheet formation and reconnection. Since future local helio-seismology observations may provide information about the sub-photospheric plasma motion, our method can be used to calculate the resulting non-forcefree coronal magnetic field configuration and plasma dynamics. This in turn can be compared with multi-wavelength observations of the solar atmosphere. Among them especially important are chromospheric and coronal emissions which allow the direct observation of deviations from the force-free configuration like chromospheric current sheets (cf. Solanki, Lagg, Woch, Krupp \& Collados (2003)).

\section{References}

Büchner, J., Nikutowski, B., \& Otto, A. 2004 in Space particle acceleration (ed. D. Gallagher), pp. 201-212, AGU monograph, Washington, 2004.

Carlsson, M. \& Stein, R.F. 2002 ApJ 572, 626-635.

Fontenla, J. M., Avrett, E. H., \& Loeser, R. 1993 ApJ 406, 319-345.

Gudiksen, B.V. \& Nordlund, A. 2002 Astrophys. J. 572, L113-L116.

Longcope, D.W. 1998 ApJ 507, 433-443.

Madjarska, M., Doyle, J.G., Teriaca, L. \& Banerjee, D. 2003 Astron. \& Astroph. 398, 775-784.

Priest, E.R., Parnel, C.E. \& Martin, S.F. 1994 ApJ 427, 459-474.

Schmidt, H.U. 1964 in Physics of Solar Flares NASA SP 50, p. 107.

Seehafer, N. 1978 Sol. Phys. 58, 215-223.

Solanki, S.K., Lagg, A., Woch, J., Krupp N. \& Collados, M. (2003) Nature 425, 692-695.

Van Driel-Gesztelyi, L. 2003 In Turbulence, Waves and Instabilities in the Solar Plasma (ed. R. Erdelyi, K. Petrovay, B. Roberts \& M. Aschwanden), pp. 281-304. Kluwer, Nato Science Series, Vol. 124.

Vernazza, J.E., Avrett, E.H. \& Loeser, R. 1981 ApJS 45, 635-725. 